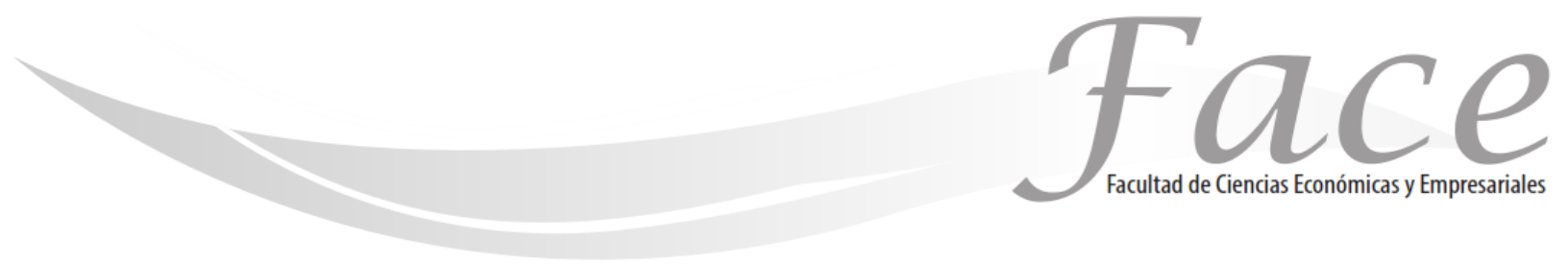

ISSN Impreso: 1794-9920 ISSN Electrónico: 2500-9338

Volumen 17-N²

Año 2017

Págs. 206 - 216

\title{
IMPACTO ECONÓMICO, SOCIAL Y FINANCIERO QUE TIENE EL MOTOTAXISMO EN LA CIUDAD DE FLORENCIA CAQUETÁ*
}

\author{
Luis Fernando Vargas ** \\ Enlace ORCID: https://orcid.org/0000-0002-6660-622X \\ Kerly Johanna Tróchez Fajardo *** \\ Enlace ORCID: https://http://orcid.org/0000-0002-2356-7013 \\ Dina Yaney Tequin Riascos **** \\ Enlace ORCID: https:// https://orcid.org/0000-0001-8355-2000
}

Fecha de Recepción: 22 de Junio 2017

Fecha de Aprobación: 24 de Agosto 2017

\section{Resumen:}

La falta de oportunidades laborales en Florencia influyó para que la informalidad creciera, el mototaxismo como problemática social; se tomó como objeto de investigación para conocer aspectos sociales, económicos y financieros que repercuten de este medio de trabajo, el objetivo fue conocer el comportamiento de diferentes variables a partir de una encuesta dirigida y aplicada a 333 personas, en su mayoría hombres con un 94\%, como resultado se conoció que esta actividad evidencia que el $91 \%$ de los encuestados tienen bajo nivel de estudio, los ingresos generados son buenos debido al precio de las tarifas que manejan para cada carrera.

Palabras clave: Mototaxismo, informal, problemática, oportunidades, educación, pobreza, comunidad.

\footnotetext{
* Este artículo es el resultado del proyecto de investigación titulado "impacto, económico, social y financiero que tiene el mototaxismo en la ciudad de Florencia, Caquetá", del Semillero de Investigación en Finanzas (SIFIN) de la Universidad de la Amazonia. Fecha de inicio: Agosto 2016, Fecha final: Mayo 2017.

** Especialista en Finanzas (Universidad del Rosario), Administrador Financiero (Universidad de lbagué Coruniversitaria); correo electrónico: Ifvargas@udla.edu.co

*** Estudiante de décimo semestre de Administración de Empresas Universidad de la Amazonia (Florencia Caquetá); Correo electrónico: fajardojohanna1@gmail.com.

${ }^{\star * \star \star}$ Estudiante de décimo semestre de Administración de Empresas Universidad de la Amazonia (Florencia Caquetá); Correo electrónico: yarick015@gmail.com.
} 


\title{
ECONOMIC, SOCIAL AND FINANCIAL IMPACT OF MOTOTAXISM IN THE CITY OF FLORENCIA CAQUETÁ - COLOMBIA.
}

\begin{abstract}
:
The lack of job opportunities in Florence influenced informality to grow, mototaxism as a social problem; it was taken as an object of investigation to know social, economic and financial aspects that have repercussions of this work environment, the objective was to know the behavior of different variables from a survey directed and applied to 333 people, mostly men with a $94 \%$, as a result it was known that this activity shows that $91 \%$ of the respondents have low level of study, the income generated is good due to the price of the fares they handle for each race.
\end{abstract}

Keywords: Mototaxism, informal, problematic, opportunities, education, poverty, community.

\section{IMPACTO ECONÔMICO, SOCIAL E FINANCEIRO DO MOTOTAXISMO NA CIDADE DE FLORENÇA - COLÔMBIA}

\section{Resumo:}

A falta de oportunidades de trabalho em Florença influenciou a informalidade para crescer, o mototaxismo como um problema social; foi tomado como objeto de investigação para conhecer aspectos sociais, econômicos e financeiros que tenham repercussões deste ambiente de trabalho, o objetivo era conhecer o comportamento de diferentes variáveis de uma pesquisa direcionada e aplicada a 333 pessoas, principalmente homens com $94 \%$, como resultado, sabia que esta atividade mostra que $91 \%$ dos respondentes têm baixo nível de estudo, a renda gerada é boa devido ao preço das tarifas que eles lidam com cada raça.

Palavras-chave: Mototaxismo, informal, problemático, oportunidades, educação, pobreza, comunidade. 


\section{INTRODUCCIÓN:}

En el presente artículo se proyecta un marco teórico donde se expone la situación actual del mototaxismo con referencias de otros países, de Colombia y de Florencia (ciudad donde se desarrolló la investigación); en la metodología se utilizó como instrumento de recolección de datos la encuesta, que se aplicó a 333 mototaxistas; para desarrollar la investigación se plantearon algunos objetivos específicos como conocer la situación actual de los mototaxistas, determinar el nivel de ingresos y analizar la incidencia de la falta de preparación académica en los mismos; estos aspectos se exponen en el análisis y discusión de este artículo, este tiene como finalidad observar, analizar y concluir la dinámica del mototaxismo como problemática social, económica y financiera en la ciudad de Florencia, se expresa de manera cuantitativa las causas y consecuencias de dicha problemática, por lo tanto para el presente estudio se usó como técnica de investigación la encuesta, "método de investigación capaz de dar respuestas a problemas tanto en términos descriptivos como de relación de variables, tras la recogida de información sistemática, según un diseño previamente establecido que asegure el rigor de la información obtenida" (Buendía , Colás, \& Hernández , 1998).

En las conclusiones se expone de forma clara y pertinente las razones por las cuales los pobladores de Florencia escogen este trabajo como una forma de sustento para su vida. De esta forma se presentan dos razones que arrojó la investigación y que se sustentan en las condiciones socioeconómicas de los Florencianos.

\section{MARCO TEÓRICO:}

En este aparte se pretende demostrar como el mototaxismo ha venido creciendo de manera rápida, como su incidencia ha marcado durante muchos años tendencia en aproximadamente 10 Departamentos del país y por supuesto en Florencia como la cuidad objetivo de esta investigación, y es que su aceptación ha sido positiva, puesto que es notorio el acelerado crecimiento de ventas de motocicletas.

Teniendo en cuenta que este es un medio de trabajo informal que acarrea inseguridad física y de salubridad para quienes hacen uso de este medio de transporte, este sigue siendo el principal servicio para trasladarse por los habitantes de Florencia; ahora bien para contextualizar sobre el tema se empieza hablando sobre el trabajo informal como un fenómeno mundial que afecta la economía, definiéndola "como la rama de las ciencias sociales que estudia los procesos de producción y distribución y el carácter de los ingresos reales" (Clement \&
Pool , 1997); "Así lo señala el informe "Perspectivas Sociales y del Empleo en el Mundo 2015", que presentó la Organización Internacional del Trabajo (OIT) y que pone en evidencia que la inseguridad va en aumento en el mercado laboral, tanto en las economías avanzadas como en las emergentes y de bajos ingresos" (Portafolio , 2015) uno de los trabajos informales que más ha cogido fuerza es el mototaxismo aunque en algunos países como la India intentan modernizar este servicio "Los mototaxis de tres ruedas, a menudo negros y amarillos, son ubicuos en India, donde los buses son pésimos, los trenes subterráneos, limitados, y los taxis son pocos y caros" (Portafolio , 2014) pero mientras en este país el mototaxismo tiene mayor aceptación; la situación en Colombia se torna muy diferente "El Ministerio de Transporte, como ente rector de la política pública de transporte, no incluyó dentro del Plan Nacional de Desarrollo (PND), la habilitación del bicitaxismo y el mototaxismo como medios de transporte público en el país." (El Colombiano , 2015).

El crecimiento del mototaxismo ha sido acelerado por tanto es difícil controlar este fenómeno y se ve reflejado en el aumento de compras de motos a nivel Nacional "En Colombia se matricularon 52.456 motos nuevas durante agosto de este año, lo cual muestra un balance de más 380.000 en lo que va corrido del año, según reportaron la Andi y Fenalco en un comunicado de prensa" (Portafolio , 2016) aunque disminuyó con respecto al mismo periodo del año anterior la cifra continúa siendo alta.

Este fenómeno hace presencia en múltiples departamentos del país "El mototaxismo es un servicio de transporte ilegal en ciudades con más de 350.000 habitantes, sin embargo hay 10 capitales de departamento en Colombia que tienen más de 4.000 mototaxis rodando en sus calles" (EI Universal , 2014), el departamento del Caquetá y principalmente su capital Florencia no es la excepción puesto que cuenta con un gran número de personas dedicadas a esta actividad informal denominada mototaxismo, esto ha generado un impacto social que es conocido como "la influencia, la huella o el efecto que la actividad de la compañía genera sobre la sociedad en la que se integra" (FORÉTICA), y es que para la ciudadanía de Florencia este es su principal medio de transporte. Según los datos obtenidos en la investigación son aproximadamente 3.000 mototaxistas, aunque esta actividad no es legal también es cierto que es el medio de subsistencia de muchas familias, por esta razón se ha tratado de llegar a una conciliación con la administración municipal; en una reunión que tuvo el alcalde de la ciudad de Florencia con el señor Manuel Urbano Polanía, representante de Moto trabajadores Asociados de Florencia, "el alcalde manifestó que quienes se dedican a esta forma de trabajo deberán demostrar que son propietarios de la motocicleta, que tienen su residencia permanente en Florencia y que realmente no tienen otra 
forma de solventar sus necesidades básicas" (La Nación , 2017 ) esto con la intención de carnetizar a los mototaxistas, teniendo en cuenta el impacto económico entiéndase como "línea de investigación que contemplan todas las aportaciones metodológicas y estudios que cuantifican en términos de renta y empleo los impactos económicos de diversas actuaciones, no solo públicas, sino también privadas", (IVIE) que este fenómeno ha tenido y que consigo ha llevado a que muchas personas de manera informal obtengan un empleo y por ende ingresos económicos como cualquier trabajo formal.

\section{METODOLOGÍA:}

Se realizó una encuesta a los mototaxistas de la ciudad de Florencia, con la que se logró obtener información referente a educación, motivos para ejercer en la informalidad, comportamiento de la ciudadanía frente al tema, tarifas entre otros y así se conoció el impacto que esta actividad ha tenido en la ciudad. Para realizar las encuestas se tomaron puntos principales como: Terminal de transportes, Universidad de la Amazonia, micro centro, diferentes clínicas que son sitios en donde se observa aglomeración de mototaxistas.

Ruta metodológica.

La investigación estuvo enmarcada en el método inductivo, "este método utiliza el razonamiento para obtener conclusiones que parten de hechos particulares aceptados como válidos" (Bernal, 2010, pág. 59), desde un análisis cualitativo y cuantitativo.

\section{Encuesta.}

El método fue aplicado a 333 mototaxistas tomados de un universo aproximado de 3.000 , se estableció mediante un formato de 12 preguntas de las cuales (7) eran cerradas y (5) abiertas, la intención era conocer la situación real de las personas que se dedican a ejercer el mototaxismo en Florencia, las preguntas se dividieron en dos ámbitos, el socioeconómico y financiero. Por último se hizo el análisis de los datos cualitativos y cuantitativos por medio de la herramienta Microsoft Excel.

\section{RESULTADOS Y DISCUSIÓN:}

La intención fundamental de esta investigación fue conocer el impacto económico, social y financiero que tiene la actividad de mototaxismo en la ciudad de Florencia, en el estudio se dan a conocer los diferentes factores socioeconómicos de las personas encuestadas; tales como: genero, edad, estrato socioeconómico, nivel de escolaridad, razones por las que decidieron ser trabajadores mototaxistas, y su lugar de origen; por otro se destacan aspectos financieros como: cuanto se ganan en un día de trabajo y precios de las diferentes carreras.

\section{Análisis socioeconómico}

\section{Gráfica N. 1}

Rangos de edad.

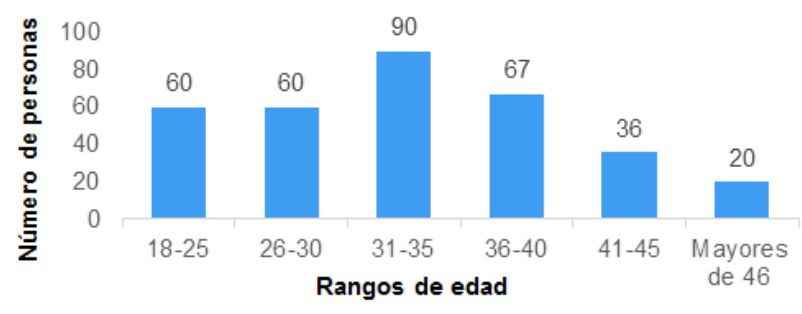

Fuente: Elaboración propia.

El total de la población encuestada fue de 333 personas pertenecientes al gremio de mototaxistas, el $94 \%$ de esta son hombres y el $6 \%$ mujeres; las personas que prestan este servicio se encuentran en un rango de edad de 18 a 30 años, (90) afirmaron tener entre 31 y 35; (103) entre 36 y 45 y (20) que pertenecen a personas mayores de 46 años (Gráfica N.1). Se puede comprobar en primera medida que la gran mayoría de personas que trabajan como mototaxistas son relativamente jóvenes, puesto que no sobrepasan los 30 años y según el estatuto juvenil de Colombia "en el país son jóvenes todas aquellas personas que tuvieran entre 14 y 26 años, y con la implementación del nuevo estatuto también lo serán quienes tengan 28 años cumplidos" (Colprensa, 2013), por otro lado se resalta que hay que 20 personas con edades superiores a los 46 , incluso algunos afirmaron tener hasta más de 50 años. 


\section{Gráfica N. 2:}

\section{Estrato socioeconómico.}

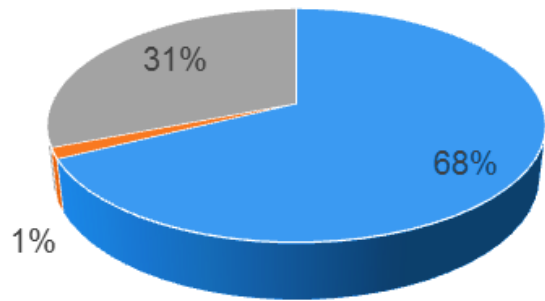

- 1 - 2 - Desplazado

Fuente: Elaboración propia.

La mayoría de las personas encuestadas argumentaron pertenecer a los estratos socio-económicos (uno y dos), es decir un $68 \%$ y $1 \%$ respectivamente, sin mencionar que un $31 \%$ pertenece a los desplazados (Gráfica N. 2), situación que es alarmante teniendo en cuenta que estas son personas que viven con limitaciones económicas bastante significativas y que esta situación puede ser fuente potencial para empezar a ejercer una actividad informal; "puesto que clasificar en los niveles 1 y 2 del Sisbén significa estar en condiciones de pobreza extrema y ser potencial beneficiario del subsidios del estado" (Caicedo, 2004, pág. 1), por otro lado "cabe tener en cuenta que, según una encuesta realizada por el DANE a 112.406 desplazados para la unidad de víctimas, al menos seis de cada diez desplazados, viven en condiciones de pobreza y que tres, de cada diez, están en la pobreza extrema" (Maya, 2015, pág. 1), es importante resaltar que "las brechas creadas por el despojo son enormes y generan desigualdad pues el 11,9 por ciento de colombianos ha sido desplazado" (Maya, 2015, pág. 1).

Gráfica N. 3

Nivel de estudios.

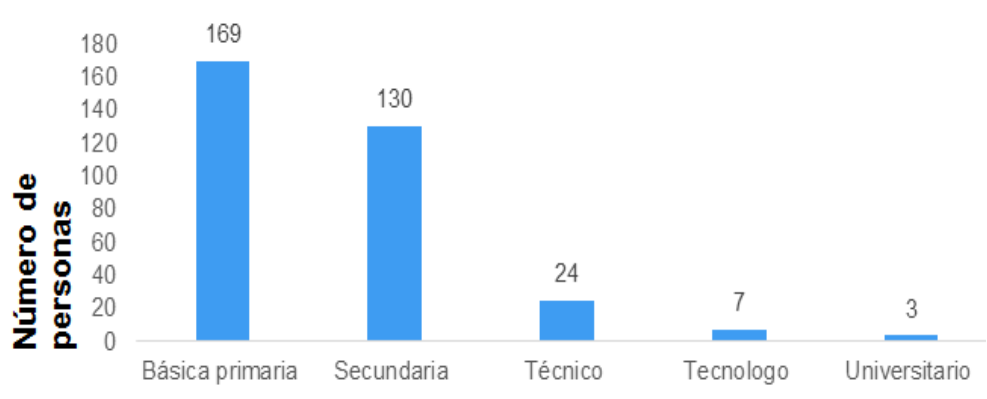

Nivel de estudios
Fuente: Elaboración propia.

En cuanto a los estudios realizados se identificó que un $51 \%$ de la población de mototaxistas realizó de manera completa los estudios correspondientes a la educación básica primaria y señalan haber iniciado sus estudios secundarios pero sin culminar, datos que son preocupantes pues es evidente una clara deserción escolar en donde "EI principal motivo por el que los jóvenes dejan de estudiar, es porque la escuela no está siendo significativa para ellos, ya que según estudios, se ha convertido en un espacio separado de la vida que no les ofrece alternativas laborales ni culturales" (Gomez, 2013 ), por otro lado un 39\% afirmó haber culminado su bachiller, un $9 \%$ ha realizado estudios técnicos y tecnólogos y solo un $1 \%$ ha cursado una carrera universitaria (Figura 3).

Es importante resaltar que la falta de preparación académica es un aspecto importante en la tasa de desempleo del país y es evidente que "el rebusque se ha convertido en la única opción laboral para millones de trabajadores sin educación superior" (Gaviria, 2010, pág. 1) y en este oficio se resalta que la mayoría de personas tienen un nivel de escolaridad muy bajo.

Gráfica N.4

¿Por qué decidió ser mototaxista?

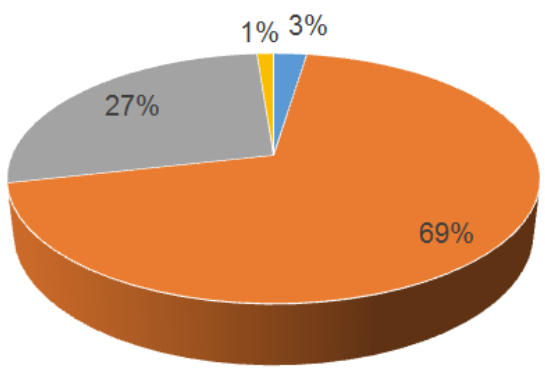

- Es una excelente forma de ganar dinero.

- Falta de empleo

- Falta de preparación académica.

w Por gusto.

Fuente: Elaboración propia.

Del total de los trabajadores mototaxistas un $69 \%$ manifestó que la falta de empleo o tasa de desempleo definida "como la relación entre la población económicamente activa (PEA) no ocupada, pero que está buscando empleo en forma activa, y la PEA total (ocupada y no ocupada)" (Urrutia, 2002) los orilló a buscar ingresos ejerciendo esta labor de mototaxistas, lo que se convierte en un claro ejemplo de informalidad definida como "todas las actividades económicas desarrolladas por los 
trabajadores y las unidades económicas que en la legislación 0 en la práctica están insuficientemente cubiertos por sistemas formales o no lo están en absoluto" (Silva, 2016). Por otro lado la falta de preparación académica con un $27 \%$ también es un factor fundamental en la decisión de buscar el mototaxismo como medio de trabajo, pues como se puede evidenciar en la (Figura 3), las personas que ejercen esta actividad tienen niveles muy bajos de estudios y es entonces como "el sector informal de una economía puede ser visto como una válvula de escape para los individuos menos educados de una sociedad" (Ochoa D. \& Ordoñez A., 2014, pág. 6) (figura 4), es importante resaltar que "la mayor parte de las personas que pertenecen al sector informal poseen un grado de escolaridad bastante bajo, el cual no supera la educación básica secundaria, lo que se convierte en una desventaja para éstos, ya que disminuyen las probabilidades de emplearse en el sector formal" (Ochoa D. \& Ordoñez A., 2014 , pág. 7) y como es notable el $90 \%$ de la población encuestada solo cuenta con el bachiller. Un porcentaje muy bajo afirmó que ejercen el mototaxismo por gusto y porque este es una excelente forma de ganar dinero lo que conlleva a factores motivacionales.

Gráfica N. 5

Lugar de origen.

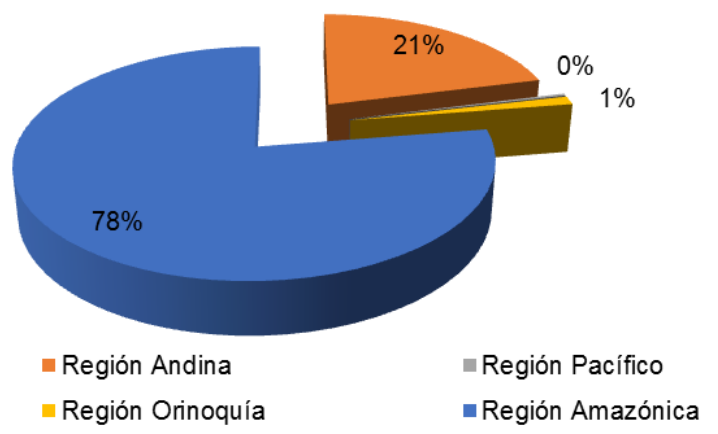

Fuente: Elaboración propia.

Se puede evidenciar que las personas que llegan a Florencia a ejercer el mototaxismo son de diferentes partes de Colombia, (Gráfica N. 5) en donde un $69 \%$ de los encuestados son de la región amazónica que comprende principalmente las personas de los diferentes municipios del Caquetá y de su capital Florencia, situación que es alarmante, pues se evidencia un alto nivel de desplazamiento, "en términos generales, es posible anotar que la dinámica del desplazamiento en Caquetá respondió, en un primer momento, a la incursión de los grupos de autodefensas en el suroccidente del departamento y a los enfrentamientos entre estos grupos y la guerrilla de las Farc por el dominio de territorios de alto valor, por la presencia de cultivos de coca y por ser corredores estratégicos para el tráfico de drogas ilícitas, armas y alimentos" (DIH, 2007, pág. 8), lo que ha provocado que "decenas de familias y poblaciones lleguen a la capital caqueteña y se ubiquen en gran mayoría en viviendas catalogados como invasores, son familias desplazadas, que a diario sufren este trauma social que impacta a las comunidades rurales obligando a dejar sus tierras y cultivos para migrar a las ciudades con la esperanza de poder sobrevivir, pero se encuentra con un escenario de escases de recursos y apoyo sociopolítico" (Narváez Rosero \& Ruiz , 2015, pág. 3), es de resaltar que las personas oriundas de Florencia también están ejerciendo este medio de trabajo informal; debido al gran nivel de desempleo que se vive constantemente en la ciudad pues "según un informe del DANE, el desempleo en el país se ubicó en $9,4 \%$, las ciudades con una alta tasa de desempleo fueron Armenia con 17,5\%, Villavicencio 12,1\%, y Florencia 11,7\%" (DANE, 2017, pág. 19).

Por otro lado también se observa que la región andina está representada por un $21 \%$ con personas que llegan principalmente de departamentos como valle del cauca, Huila, Tolima, Cauca, Bogotá, Quindío entre otros y la principal razón, la falta de empleo en sus lugares de origen, pero ¿Por qué decidieron llegar a Florencia donde la situación de empleo es peor que en muchos de estos departamentos? Esta pregunta será resuelta más adelante.

Gráfica N. 6

Tiempo que lleva ejerciendo el Mototaxismo.

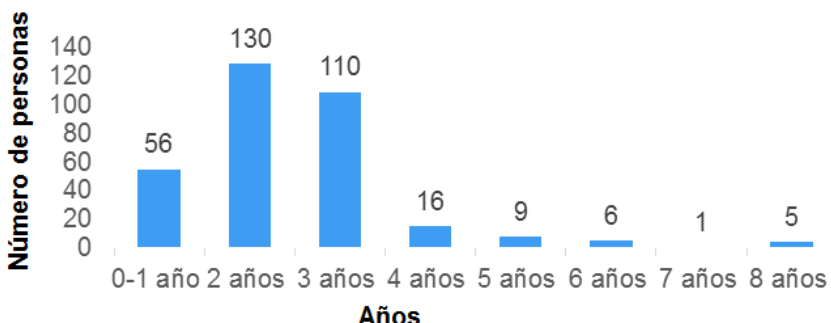

Fuente: Elaboración propia.

Hablando en términos del tiempo que se lleva ejerciendo como mototaxista (56) personas de la población encuestada lleva (1) año en la informalidad, (130) y (110) entre (2) y (3) años respectivamente y 37 personas aseguraron llevar en esta actividad entre (4) y hasta (8) años. (Gráfica N. 6), este tiempo es preocupante, pero para muchos de ellos esto se convirtió en un estilo de vida, además, que en el país se está haciendo más difícil conseguir empleo puesto que últimamente en "Colombia no hay una relación entre la formación superior profesional, 
técnica y tecnológica y el mercado laboral" (Morales, 2016, pág. 1) y por supuesto resaltando el escaso nivel de escolaridad de estas personas.

Gráfica N. 7.

¿Por qué vio en Florencia una ciudad para trabajar como mototaxista?

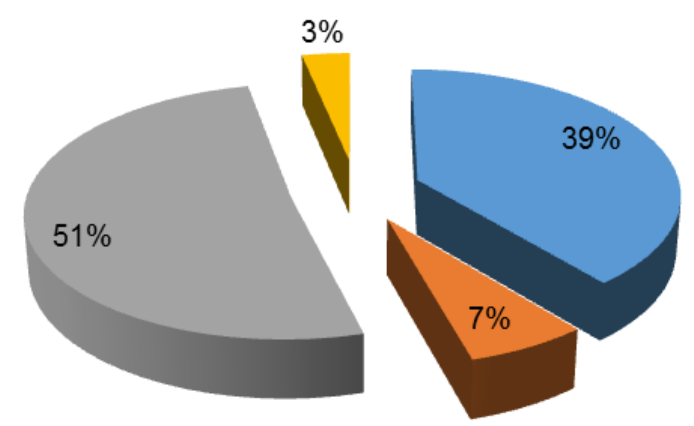

- El servicio de mototaxi creció rápidamente en la cuidad. - Había muchas incomodidades con el servicio de bus y taxis. - La comunidad de la ciudad de Florencia acogió bien el servicio. NS/NR

Fuente: Elaboración propia.

Hablando en términos del tiempo que se lleva ejerciendo como mototaxista (56) personas de la población encuestada lleva (1) año en la informalidad, (130) y (110) entre (2) y (3) años respectivamente y 37 personas aseguraron llevar en esta actividad entre (4) y hasta (8) años. (Gráfica N. 6), este tiempo es preocupante, pero para muchos de ellos esto se convirtió en un estilo de vida, además, que en el país se está haciendo más difícil conseguir empleo puesto que últimamente en "Colombia no hay una relación entre la formación superior profesional, técnica y tecnológica y el mercado laboral" (Morales, 2016, pág. 1) y por supuesto resaltando el escaso nivel de escolaridad de estas personas.
Gráfica N. 8

¿Cuál es su horario de trabajo?

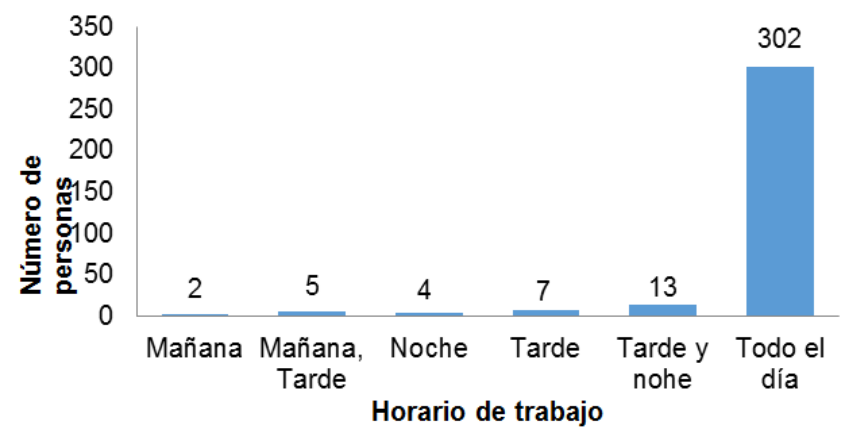

Fuente: Elaboración propia.

Las personas que ejercen el mototaxismo tienen la posibilidad de decidir su horario de trabajo, se puede evidenciar que (302) de las (333) personas encuestadas trabajan durante todo el día, es decir con horarios que propasan las 8 horas, incluso en comentarios se decía que su jornada laboral empezaba desde las 5 de la mañana y terminaba a las 9 de la noche y que sus ingresos diarios eran buenos para un horario tan extenso, claro y es que como se muestra en la (Gráfica N. 10), las personas que manejan este horario llegan a obtener ingresos superiores a $\$ 30.000$ pesos al día. Por otro lado (31) mototaxistas argumentaron que prefieren horarios donde han notado que hay más afluencia de personas como lo es en la tarde y algunas veces después de la $6 \mathrm{pm}$. 


\section{Gráfica N. 9}

¿Cómo maneja los diferentes controles que ejerce la administración pública para la seguridad de los motociclistas? (ejemplo: uso de cascos reglamentarios, chalecos, placas).

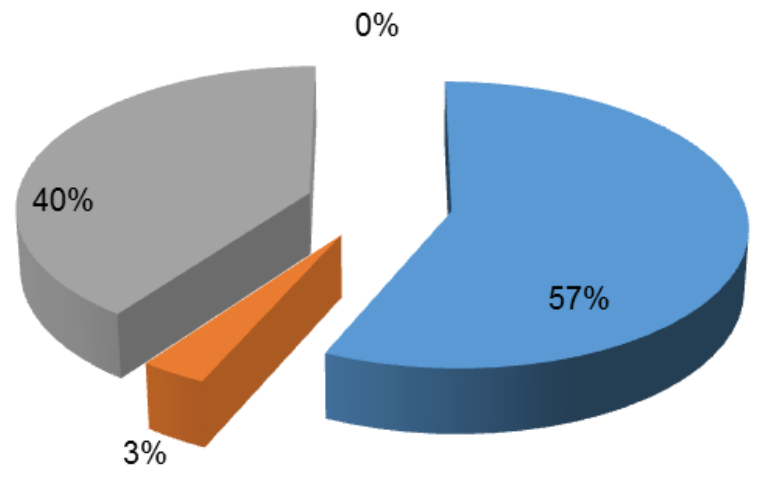

- cumplo con algunas normas y evito encuentros con la policía. - hago caso omiso y continuo con mi trabajo.

- Me adapto con facilidad y cumplo las normas

- Otro.

\section{Fuente: Elaboración propia.}

Es notorio que los mototaxistas en un gran porcentaje no hacen uso de los sistemas de seguridad para él y los pasajeros, un $57 \%$ cumple con algunas normas, pero infringen una muy importante, que es el seguro vigente SOAT; situación que es peligrosa porque "Pese a que los usuarios argumentan varios beneficios, los mototaxis también ofrecen varios riesgos, sin embargo, el principal es que llegado el caso que se presente un accidente de tránsito, nadie responderá por la integridad ni los daños del pasajero, pues estas motos no cuentan con un seguro especial como sí lo tienen los vehículos de transporte público legal" (NACIÓN, 2017, pág. 2). Otro porcentaje asegura que se adapta con facilidad y que cumple con las normas, cosa que no es tan cierta, pues se pudo observar un bastante desinterés de ellos en hacer unos de los cascos reglamentarios, teniendo en cuenta que "La utilización del casco es una norma general consagrada expresamente por el Código Nacional de Tránsito para quienes transiten en motocicletas, bicicletas y similares. El casco es un dispositivo de seguridad cuyo fin es la protección de la cabeza contra golpes, sin impedir de manera alguna la visibilidad de quien lo utiliza" (Llanos, 2004, pág. 1) y por ultimo un $3 \%$ aseguró que simplemente hace caso omiso; pues para ellos lo más importante es poder trabajar.

Análisis financiero
Gráfica N. 10

Aproximadamente, ¿cuánto es el producido en un día de trabajo?

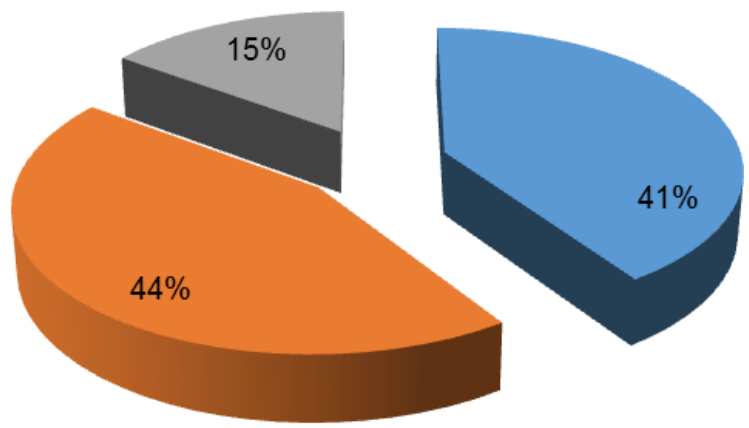

Fuente: Elaboración propia.

Hablando en términos financieros, en cuanto al producido en un día de trabajo se observa en la (Grafica N. 10), que este tipo de negocio informal tiene un lucro bastante significativo para las personas que lo ejercen; si bien, se puede notar que un $44 \%$ de los encuestados asegura que en un día de trabajo pueden obtener ingresos de $\$ 26.000$ a $\$ 30.000$ pesos, un $41 \%$ entre $\$ 15.000$ y $\$ 25.000$ y solo un $15 \%$ afirmaron ganar entre $\$ 31.000$ y $\$ 36.000$ pesos. Tomando el primer grupo, se puede establecer que multiplicando la ganancia diaria por los 30 días del mes, se obtienen ingresos mensuales de $\$ 780.000$ y $\$ 900.000$ pesos respectivamente, es de resaltar que esta ganancia es fuera de gastos en gasolina y la cuota diaria cuando no son los propietarios de las motos.

En este caso se puede concluir que el mototaxismo en Florencia está generando en la mayoría de los casos ingresos cercanos o superiores a un Salario Mínimo Legal Vigente (SMLV), teniendo en cuenta que "el salario mínimo para este 2017 será de $\$ 737.717$, el incremento de auxilio de transporte del 7\% hasta $\$ 83.140$ " (Portafolio, 2017, pág. 1) lo que desarrolla un alto nivel motivacional para ejercer en la informalidad y este caso en el popular mototaxi. 
Grafica N. 11

Clasifique las tarifas de las carreras desde la más baja hasta la más alta.

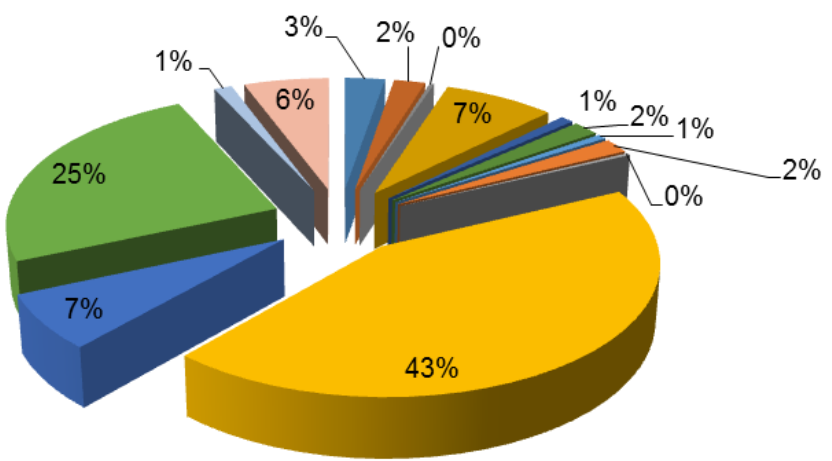

$=1.500-2.000 \quad 1.000-3.000 \approx 1.000-5.000 \backsim 1.500-3.000$

$=1.500-4.000=1.500-5.000=1.000-2.000=1.000-3.000$

$=1.500-8.000=2.000-3.000=2.000-4.000=2.000-5.000$

$=3.000-4.000-3.000-5.000$

Fuente: Elaboración propia.

Es evidente que en Florencia un $43 \%$ de los mototaxistas están manejando las tarifas de las carreras a partir de $\$ 2.000$ pesos la más baja y $\$ 3.000$ la más cara, un $25 \%$ asegura que cobra la carrera más económica y la más cara entre $\$ 2.000$ y $\$ 5.000$ pesos respectivamente, por otro lado un $32 \%$ de los encuestados manejan tarifas desde $\$ 1.000$ la económica y $\$ 5.000$ la más costosa. Estas diferencias están enmarcadas de acuerdo al lugar donde se dirige la persona y el horario en que se hace la carrera, pues según los mototaxistas; las carreras en horas de la noche son más costosas. Ahora si se hace un comparativo en cuanto al pasaje en un bus, en Florencia el pasaje en el transporte público bus cuesta 1.400 y en taxi 4.800 pesos, el primero es más económico comparado con una carrera en mototaxi; pero resulta que las rutas no llegan a todos los barrios de la capital caqueteña, en cuanto a servicio de taxi, este es más costoso que un pasaje en moto, es ahí donde se puede comprender por qué el negocio resulta ser bastante lucrativo, resulta que últimamente a los habitantes de Florencia le es más fácil y cómodo hacer uso de los mototaxis.

\section{CONCLUSIONES:}

De la investigación se puede concluir que el mototaxismo es consecuencia de dos factores muy importantes: el primero que el nivel de educación de los mototaxistas es muy bajo, puesto que el $51 \%$ de la población encuestada solo culminó sus estudios primarios, el segundo factor a tener en cuenta es el desempleo que está relacionado con el bajo nivel de escolaridad debido a que un empleo formal en una empresa es menos asequible para la población con bajo nivel de estudio.

El mototaxismo ha generado un gran impacto porque los habitantes de la ciudad de Florencia prefieren hacer uso de este medio de transporte debido a que el servicio de bus no llega a todas los lugares de la ciudad; mientras que los mototaxistas les están brindando un servicio puerta a puerta, este resulta ser un poco más costoso que el pasaje en bus, puesto que el pasaje en bus cuesta $1.400 \mathrm{y}$ el servicio de mototaxi entre 2.000 y 3.000 pesos, pero genera un beneficio adicional que sería el mismo que ofrece un taxi pero este maneja una tarifa más alta.

Los mototaxistas ya se acostumbraron a tener jornadas de trabajo extensas, debido a que el $91 \%$ de la población encuestada prefieren trabajar durante todo el día, pues para ellos entre más temprano salgan y más tarde terminen más van a ganar, como hay otros que prefieren filtrar su horario, es decir salen en horas y pico (12:00 m.d. y 6:00 p.m.) y lugares específicos donde tienen conocimiento que hay mayor acumulación de pasajeros.

El mototaxismo se ha convertido en una problemática social, puesto que la administración pública no ha sensibilizado a la población en cuanto a los riesgos que repercuten al hacer uso de este medio de transporte informal, puesto que es la misma quien lo ha aceptado y hace uso del mismo; los habitantes de la capital caqueteña prefieren acceder a este servicio aun cuando saben y conocen la inseguridad que este ha provocado (constantes accidentes, hurtos, caos y revueltas); lo que esta no ha comprendido es que de manera indirecta está ayudando a que este fenómeno social aumente significativamente, pues el solo hecho de hacer uso de este servicio una vez al día contribuye a que esta problemática siga creciendo; es importante que las personas comprendan que en manos de ellos está la solución a que este fenómeno disminuya o se erradique de forma definitiva.

Por último como se evidenció en los resultados de la encuesta aunque los ingresos al desempeñar la labor de mototaxistas son relativamente altos, también se identificó que las personas que la ejercen tienen un nivel de estudios bajo, por tanto es importante recomendar a esta población que parte de la falta de oportunidades para desempeñar una labor diferente es prepararse mejor académicamente, accediendo a las diferentes entidades públicas que oferten programas académicos 


\section{REFERENCIAS:}

El Universal . (02 de Diciembre de 2014). Obtenido de El Universal.

http://www.eluniversal.com.co/colombia/losmototaxistas-se-tomaron-10-capitales-decolombia-178487

Portafolio . (08 de Agosto de 2014). Obtenido de Portafolio http://www.portafolio.co/internacional/indiamodernizan-mototaxismo-51042

El Colombiano . (12 de Mayo de 2015). Obtenido de El Colombiano

http://www.elcolombiano.com/colombia/encolombia-no-se-legalizara-el-mototaxismomintransporte-IJ1902659

Portafolio . (18 de Mayo de 2015). Obtenido de Portafolio : http://www.portafolio.co/internacional/informalidad -domina-relaciones-laborales-mundo-26832

Portafolio . (14 de Septiembre de 2016). Obtenido de Portafolio

http://www.portafolio.co/economia/cifras-delsector-de-las-motos-en-colombia-2016-500292

La Nación . (13 de Julio de 2017 ). Obtenido de La Nación : http://www.lanacion.com.co/2017/07/13/alcaldiaflorencia-caracterizara-mototaxistas/

Angulo, Y. F. (2011). El mototaxismo. Florencia .

Bernal, C. A. (2010). Metodología de la Investigación. Mexico: PEARSON.

Buendía , L., Colás, P., \& Hernández , F. (1998). Métodos de Investigación en Psicopedagogía. Madrid: McGraw-Hill.

Caicedo, F. (23 de ENERO de 2004). SISBÉN DICE QUE LOS POBRES SON MÁS DE LOS QUE SE CREE. EL TIEMPO, pág. 1

Clement , N. C., \& Pool , J. C. (1997). Economía: Enfoque América Latina. McGraw-Hill.

Colprensa. (1 de MAYO de 2013). Nuevo estatuto juvenil considera jóven a una persona entre 14 y 28 años. eICOLOMBIANO, pág. 1.
DANE. (30 de JUNIO de 2017). Boletín Técnico Cominicación Informativa . Recuperado el 17 de SEPTIEMBRE de 2017, de https://www.dane.gov.co/files/investigaciones/bol etines/ech/ech/bol_empleo_may_17.pdf

DIH, B. d. (2007). Diagnóstico Departamental Caquetá. Florencia.

FORÉTICA, L. R. (s.f.). FORÉTICA, LIDERAZGO RESPONSABLE. Recuperado el 23 de MAYO de 2018, de http://www.foretica.org/tematicas/medicionimpacto-social/

Gaviria, A. (07 de MARZO de 2010). El problema del empleo. EL ESPECTADOR, pág. 1.

Gomez, B. C. (03 de JUNIO de 2013 ). COLPRENSA. Recuperado el 16 de SEPTIEMBRE de 2017, de http://www.elcolombiano.com/historico/en_colomb ia_el_50_por_ciento_de_los_jovenes_abandona_ la_educacion_secundaria-FBEC_245011

IVIE. (s.f.). Ivie, Áreas de investigación. Recuperado el 23 de MAYO de 2018, de http://www.ivie.es/es_ES/investigacion/proyectos/i mpacto-economicol

Llanos, S. M. (02 de OCTUBRE de 2004). EL CASCO Y EL CHALECO SON PRIORIDAD. EL TIEMPO.

Maya, E. (16 de FEBRERO de 2015). Tres de cada diez desplazados están en pobreza extrema. EL TIEMPO, pág. 1.

Morales, E. (2016). ¿Por qué es tan difícil conseguir empleo en Colombia? SEMANA.

NACIÓN, L. (2 de ABRIL de 2017). Florencianos prefieren el mototaxi. LA NACIÓN.

Narváez Rosero, N., \& Ruiz , L. M. (03 de ABRIL de 2015). Las causas y consecuencias del desplazamiento forzado en Florencia Caquetá. Recuperado el 17 de SEPTIEMBRE de 2017, de http://grupocolaborativo172.blogspot.com.co/ 
Ochoa D. \& Ordoñez A. (2014). INFORMALIDAD EN COLOMBIA. CAUSAS, EFECTOS $Y$ CARACTERÍSTICAS DE LA ECONOMÍA DEL REBUSQUE. Universidad ICESI.

Ochoa D. \& Ordoñez A. (2014). INFORMALIDAD EN COLOMBIA. CAUSAS, EFECTOS $Y$ CARACTERISTICAS DE LA ECONOMÍA DEL REBUSQUE. Universidad ICESI.

OIT . (2004). INFORMALIDAD EN COLOMBIA. CAUSAS, EFECTOS Y CARACTERÍSTICAS DE LA ECONOMÍA DEL REBUSQUE.

Portafolio. (2017). SALARIO MINIMO EN COLOMBIA 2017. PORTAFOLIO.

Sampieri Hernández , R., Collado Fernández , C., \& Lucio Baptista, P. (2003). Metodología de la Investigación. Mexico.

Silva, O. (2016). Utrein Iberoaméricana. Recuperado el 17 de SEPTIEMBRE de 2017, de http://www.utrein.org/definicion.html

Urrutia. (Agosto de 2002). Banco de la república. Recuperado el 16 de SEMTIEMBRE de 2017, de http://www.banrep.gov.co/documentos/publicacio nes/pdf/Ago02.pdf

Valencia, A. M. (8 de AGOSTO de 2016). A LA ORILLA DEL RIO. Recuperado el 16 de SEPTIEMBRE de 2017, de http://alaorilladelrio.com/2016/08/08/encaqueta-el-413-de-los-habitantes-son-pobres/ 